\title{
Oncofertility Approach in Females With Leukemia From the Reproductive Perspective
}

\author{
Ana Kisovar, Eda Vrtačnik Bokal \\ Department of Human Reproduction, University Medical Centre Ljubljana, Slovenia
}

\begin{abstract}
Leukemia is one of the most common types of cancer in young people, with 5 -year survival rates ranging from $54 \%$ to $94 \%$. Due to gonadotoxic treatment, the chances of subsequent pregnancies in leukemia survivors are generally low. To date, several methods for fertility preservation have been presented, but options for this patient group remain limited. A multidisciplinary oncofertility approach with tight coordination between hemato-oncologists and reproductive specialists is critical for maintaining a high standard of oncofertility care to increase chances for fertility preservation in females with leukemia.
\end{abstract}

Keywords: fertility preservation, oncofertility counseling, oncofertility approach, females with leukemia, gonadotoxicity.

\section{INTRODUCTION}

Leukemia is one of the most common types of cancer in young people, representing $28 \%$ of all childhood cancers, $13 \%$ of cancers in adolescents, and $3 \%$ of all newly diagnosed cancers in females. ${ }^{1}$ The 5 -year survival rates vary between $54 \%$ and $94 \%$, depending on the type of leukemia and the patient's age at the time of diagnosis. The most common types of leukemia in children and adolescents are acute lymphocytic leukemia (ALL) and acute myeloid leukemia (AML). However, chronic myeloid leukemia (CML) and chronic lymphocytic leukemia (CLL) can also occur in these age groups. ${ }^{1}$ Each of these hematological malignancies can adversely affect fertility due to the inherent characteristics of the disease itself, the gonadotoxic potential of specific anti-cancer therapy and the patient's age at diagnosis. ${ }^{2}$ Taking all of these aspects into account, female leukemia survivors have significantly compromised chances of subsequent pregnancies. ${ }^{3}$

Despite the strong potential of anti-cancer therapy to impair fertility, along with the strong impact of infertility on quality of life, the issue of fertility preservation in cancer patients has still not been completely clarified and routinely discussed in clinical practice. ${ }^{4,5}$ Therefore, the term oncofertility was introduced, representing a new interdisciplinary field at the crossing of oncology and reproductive medicine, to promote fertility preservation in young cancer patients. ${ }^{6}$ Based on current clinical experience, the oncofertility approach in leukemia is particularly demanding. Therefore, in this article, we focus on the current multidisciplinary challenges to improve fertility preservation outcomes in these patients and encourage communication between hemato-oncologists and reproductive specialists.

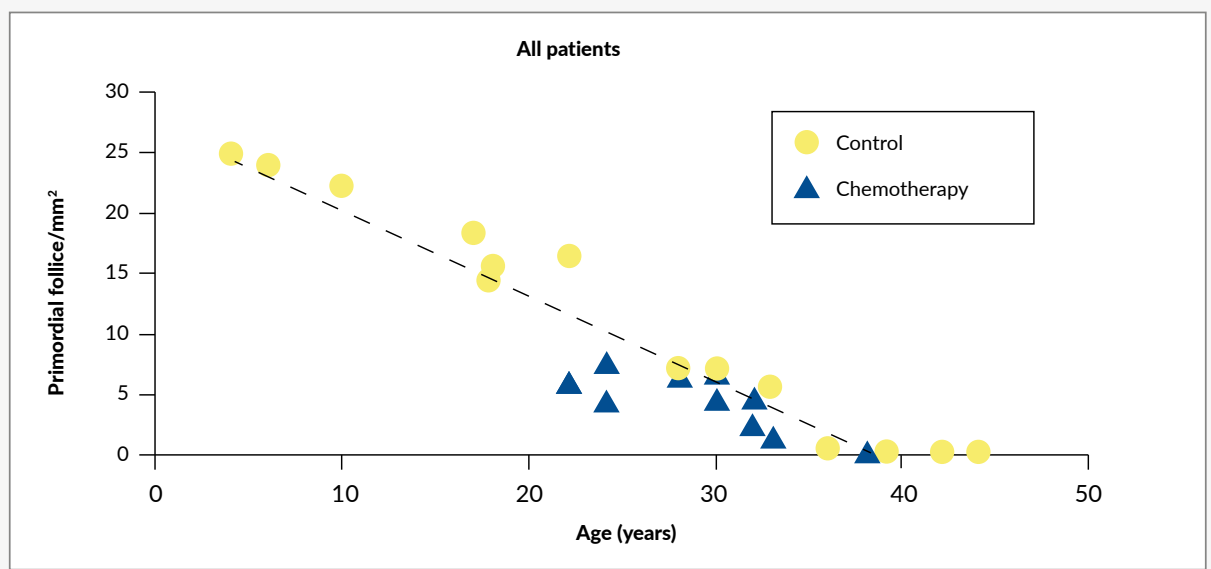

Corresponding author:

Ana Kisovar, MD

Department of Human Reproduction, University Medical Centre Ljubljana, Slovenia

E-Mail: ana.kisovar@gmail.com

DOI: $10.36000 /$ hbT.OH.2020.04.015 ISSN: 2673-2092 (Print) and 26732106 (Online)

This article was published on July 10, 2020

Kisovar et al. Oncofertility Approach in Females With Leukemia From the Reproductive Perspective. healthbook TIMES Onco Hema 2020;(4):10-17.
Figure 1. Physiologic versus an accelerated decrease of ovarian reserve. Adapted from Oktem et al. 2007. ${ }^{10}$ 


\section{GONADOTOXICITY}

\section{Mechanisms and risk assessment}

Females are born with around 1 million primordial follicles, and by the time of puberty, only about 400,000 of them remain. ${ }^{7}$ In each menstruation cycle, multiple follicles enter the maturing pool. Due to the follicle-stimulating hormone (FSH) stimulation, one of them becomes the dominant follicle, which produces estradiol triggering a luteinizing hormone (LH) surge followed by ovulation. The remaining follicles in the maturing pool undergo atresia and apoptosis, leading to a decline in the number of primordial follicles with increasing age. ${ }^{8}$ This physiological decrease of ovarian reserve can be significantly accelerated by gonadotoxic anti-cancer treatment. Chemotherapy actively targets dividing cells and induces apoptosis in granulosa cells essential for the growing follicles, leading to the destruction of mature ovarian follicles. Moreover, chemotherapy can also cause apoptosis of nongrowing primordial follicles, ovarian atrophy and cortical fibrosis. ${ }^{9}$ All these processes can result in either premature menopause in case of moderate depletion of primordial follicles, or even in acute ovarian failure in case of almost complete depletion (Figure 1). ${ }^{7}$

There is still a significant inconsistency in monitoring gonadotoxicity, mostly due to different availability of data in medical records, type of laboratory tests, the variability of follow-up methods, and lack of control groups. ${ }^{11}$ Additionally, different classifications to assess the risk of gonadotoxicity exist for different agents. One of the most commonly used classifications based on the prevalence of chemotherapyinduced amenorrhea was proposed by the Livestrong organization (Table 1). ${ }^{12}$

Table 1. Risk assessment for gonadotoxicity. Adapted from Livestrong. $2007 .{ }^{12}$

\begin{tabular}{|c|c|c|}
\hline High risk & $\begin{array}{l}>80 \% \text { of women develop } \\
\text { posttreatment amenorrhea }\end{array}$ & \multirow{2}{*}{$\begin{array}{l}\text { Fertility preservation } \\
\text { counseling should be } \\
\text { undertaken prior to } \\
\text { treatment. }\end{array}$} \\
\hline Intermediate risk & $\begin{array}{l}30-70 \% \text { of women develop } \\
\text { posttreatment amenorrhea }\end{array}$ & \\
\hline Low risk & $\begin{array}{l}<20 \% \text { of women develop } \\
\text { amenorrhea posttreatment }\end{array}$ & \multirow{2}{*}{$\begin{array}{l}\text { Fertility preservation } \\
\text { counseling should be } \\
\text { undertaken before or after } \\
\text { the treatment. }\end{array}$} \\
\hline Very low/no risk & Negligible effect on menses & \\
\hline Unknown risk & & $\begin{array}{l}\text { Fertility preservation } \\
\text { options should be dis- } \\
\text { cussed. }\end{array}$ \\
\hline
\end{tabular}

Chemotherapy-induced amenorrhea is typical in cancer survivors, where menstruation usually resumes within two years, however, these women may still have decreased ovarian reserve. ${ }^{13,14}$ Among other frequently used indicators are pregnancy and birth rates, infertility measured as an inability to conceive after one year of attempting, acute ovarian failure, premature menopause, and gonadal insufficiency. ${ }^{11}$ In this regard, anti-Müllerian hormone (AMH) very accurately correlates with the antral follicle count (defined as the number of follicles in the ovary measuring $2-10 \mathrm{~mm}$ in diameter).$^{15}$ It can, therefore, predict decreased ovarian reserve earlier than other laboratory markers. It is possible to determine the concentration of $\mathrm{AMH}$ in prepubertal as well as postpubertal females. ${ }^{16}$

\section{Systemic anti-cancer therapy}

Chemotherapeutic agents can impair fertility by inducing prenatal loss of oogonia, direct loss of primordial follicles, accelerated activation of primordial follicles, follicular atresia, damage to the ovarian stromal tissue or vasculature, and inflammation. ${ }^{17}$ Importantly, understanding the mechanisms by which cancer therapies cause gonadal impairment may lead to the development of neoadjuvant cytoprotective pharmacotherapy, capable of protecting the ovary without compromising the efficacy of the anti-cancer therapy. ${ }^{18}$ Table 2 presents standard anti-cancer agents for leukemia in the target age group, and their known gonadotoxic mechanisms. ${ }^{19-23}$

In general, the reproductive ability of girls and young women treated with conventional chemotherapeutic protocols alone for ALL and AML is mostly protected. ${ }^{38}$ Low-dose regimens have demonstrated better fertility outcomes in the treatment of Hodgkin lymphoma, showing that those options could also be applied for leukemia patients. ${ }^{39}$ Less is known of infertility in CLL and CML patients since the gonadotoxic effect of protein kinase inhibitors is less well-established. ${ }^{40}$ Similarly, no potential risks are known for other immunotherapy strategies, such as chimeric antigen receptor (CAR) T-cell therapy and donor lymphocyte infusions. ${ }^{41}$ However, patients receiving these treatments are often heavily pretreated, meaning their fertility is likely already impaired by previous regimens. Clearly, a better understanding of the gonadotoxic mechanisms of novel chemotherapeutics and combination therapies is needed not only to protect against iatrogenic depletion of ovarian follicles but also to prevent endocrine disruptions. ${ }^{42}$

\section{Radiotherapy}

Although total body irradiation for hematopoietic stem cell transplantation (HSCT) is rarely used nowadays, cranial radiotherapy for metastatic disease can cause disorders on the hypothalamic-pituitary axis, and pelvic radiotherapy can severely impair ovarian function by causing depletion of germ cells and reduction in the production of sex hormones. ${ }^{43-45}$ Several radiation oncologists recommend fractionated radiotherapy whenever possible to minimize direct and indirect damage to the ovaries. ${ }^{4-49}$ 


\section{healthbook TIMES Oncology Hematology}

Table 2. Mechanism and risk assessment for individual systemic anti-cancer agents.

$H R$, high risk; IR, intermediate risk; LR, low risk; UR, unknown risk; VLR, very low/no risk.

\begin{tabular}{|c|c|c|}
\hline Systemic anti-cancer therapy & Degree of risk & Mechanism and indication of gonadotoxicity \\
\hline Alkylating agents & & $\begin{array}{l}\text { Formation of cross-links with DNA, subsequent inhibition of DNA synthesis and function, leading } \\
\text { to apoptosis of human primordial follicles. }{ }^{24}\end{array}$ \\
\hline Cyclophosphamide & $\mathrm{HR}$ & $\begin{array}{l}\text { In cancer patients, cyclophosphamide equivalent dose was associated with significantly reduced } \\
\text { pregnancy rates and live births at the doses higher than } 11.3 \mathrm{~g} / \mathrm{m}^{2}(\mathrm{HR}=0.85[95 \% \mathrm{Cl} \text { : } \\
0.74-0.98]) .{ }^{25} \text { Effects are dose-, duration-, and age-dependent, but not cycle-dependent. }{ }^{18}\end{array}$ \\
\hline Anthracyclines & & $\begin{array}{l}\text { Direct loss of primordial follicles by inhibition of topoisomerase II, leading to DNA breaks and by } \\
\text { inducing oxidative stress. }{ }^{26}\end{array}$ \\
\hline Doxorubicin & IR & $\begin{array}{l}\text { In in vitro models of human ovaries, premature ovarian aging was found as a consequence of a } \\
\text { complicated mechanism involving the germ- as well as non-germ cell components of the ovary. }{ }^{27}\end{array}$ \\
\hline Daunorubicin & LR & $\begin{array}{l}\text { In } 15 \% \text { of cancer patients receiving daunorubicin among other anti-cancer agents premature } \\
\text { ovarian failure was observed. } .^{28}\end{array}$ \\
\hline Antimetabolites & & $\begin{array}{l}\text { Interference with DNA, RNA, thymidylate and purine synthesis with no damage in human follicles } \\
\text { DNA. }{ }^{29}\end{array}$ \\
\hline Methotrexate & LR & $\begin{array}{l}\text { None of the patients with breast cancer under the age of } 34 \text { years have experienced amenorrhea } \\
\text { after combination treatment with mehotrexate. } .^{30}\end{array}$ \\
\hline Cytarabine & UR & We found no specific data. \\
\hline Fludarabine & UR & We found no specific data. \\
\hline Vinca alkaloids & & $\begin{array}{l}\text { Inhibition of polymerization of tubulin and disruption of assembly of microtubules during mitosis, } \\
\text { leading to cell death with no damage on DNA in human follicles. } .^{29}\end{array}$ \\
\hline Vincristine & LR & $\begin{array}{l}\text { In female mice, it has been demonstrated that vincristine, administered at the recommended } \\
\text { dose, has gonadotoxic effects by damaging growing follicles without depleting primordial } \\
\text { follicles. }^{31}\end{array}$ \\
\hline Protein kinase inhibitors & & $\begin{array}{l}\text { Follicular atresia, caused by inhibition of several signaling pathways, which play an important role } \\
\text { in the placental and gonadal development. }{ }^{32}\end{array}$ \\
\hline Imatinib & VLR & $\begin{array}{l}\text { In females, ovarian failure is not a recognized complication. However, in a } 28 \text {-year-old female } \\
\text { CML patient, the development of primary ovarian deficiency was reported after two years of } \\
\text { treatment. }\end{array}$ \\
\hline Nilotinib & UR & $\begin{array}{l}\text { In female mice, a statistically significant decrease was demonstrated in the total follicle numbers } \\
\text { in the nilotinib group when compared with the control group }(268 \pm 110 \text { vs } 170 \pm 60 ; p=0.03) .^{34}\end{array}$ \\
\hline Dasatinib & UR & $\begin{array}{l}\text { In female rats, dasatinib was observed to be teratogenic, extensively distributed in maternal } \\
\text { tissues, and secreted into milk. }{ }^{35}\end{array}$ \\
\hline Ibrutinib & UR & We found no specific data. \\
\hline Bosutinib & UR & We found no specific data. \\
\hline Ponatinib & UR & We found no specific data. \\
\hline Idelalisib & UR & We found no specific data. \\
\hline \multicolumn{3}{|l|}{ Immunotherapy } \\
\hline Rituximab & VLR & No direct impact on the ovary and fertility..$^{40}$ \\
\hline CAR T-cell therapy & UR & We found no specific data. \\
\hline Donor lymphocyte infusion & UR & We found no specific data. \\
\hline $\mathrm{BCL} 2$ antagonist of cell death & & $\begin{array}{l}\text { In vivo and in vitro studies have shown induced apoptosis within the ovary through several } \\
\text { mechanisms. }{ }^{36}\end{array}$ \\
\hline Venetoclax & UR & We found no specific data. \\
\hline \multicolumn{3}{|l|}{ Others } \\
\hline Asparaginase & VLR & In female hamsters, no toxic effects on ovaries were detected. ${ }^{37}$ \\
\hline
\end{tabular}




\section{Oncofertility in Females With Leukemia}

\section{FERTILITY PRESERVATION}

To date, several different strategies for fertility preservation have been applied to cancer patients. ${ }^{46}$ The technique selection should depend on the treatment plan, the age, the risks and efficacy, the individual preference of the patient as well as costs and accessibility. ${ }^{47}$ Nevertheless, the options for the distinct population of females with leukemia are still limited.

\section{Prepubertal girls}

Unfortunately, the most conventional methods, such as embryo and oocyte cryopreservation, are not suitable for prepubertal girls due to the inactive hypothalamic-pituitaryovarian axis, leaving them with only limited options for fertility preservation, such as ovarian tissue cryopreservation, oocyte maturation in vitro, and other novel methods.

\section{Ovarian tissue cryopreservation}

Ovarian tissue cryopreservation, followed by autotransplantation, is the most discussed currently available option for prepubertal female cancer survivors, which can restore not only reproductive but also endocrine ovarian function. ${ }^{46}$ Here, a part of the cortical ovarian tissue is surgically excised, cryopreserved, and transplanted back when the cancer is cured and the pregnancy desired. Corresponding pregnancy rates after ovarian transplantation in postpubertal females are $23-37 \%$, while data on outcomes in prepubertal cryopreserved ovarian tissue is limited to a first successful live birth in $2015 .^{48,49}$ Furthermore, there is a high risk of ovarian metastases in leukemia, which were found in more than $50 \%$ of patients. ${ }^{50}$ That is why ovarian tissue cryopreservation with auto-transplantation should still be considered as an experimental technique in this setting. ${ }^{50}$ Nevertheless, the first case of delivery in a leukemia patient has been reported, in which they evaluated ovarian tissue for leukemia cells contamination prior to autotransplantation. ${ }^{51}$ Some authors conclude this procedure should still be performed, considering the progression of novel techniques, such as in vitro maturation and artificial ovary. ${ }^{52-54}$ However, the patients should be thoroughly informed about the possible risks and the potential futility of this promising method. ${ }^{52}$

\section{Oocyte maturation in vitro}

One step further from ovarian tissue cryopreservation is in vitro maturation of oocytes, which represents a possible alternative to ovarian tissue cryopreservation for cancer patients with a high risk of ovarian metastases. ${ }^{48}$ In this method, oocytes are extracted either in vivo or ex vivo from the excised ovarian tissue, then cultured in vitro for up to 48 hours before in vitro fertilization (IVF) or cryopreservation..$^{53}$ Although the success rates after a fresh transfer are comparable to the traditional IVF, the success rates after cryopreservation are still controversial..$^{55-58}$ The first live birth after in vitro maturation and cryopreservation was in 2020.59 Nevertheless, due to existing controversies, von Wolff et al. (2018) recommended that the method should be limited to women with a very high antral follicle count and without time to perform gonadotropin stimulation. ${ }^{60}$

\section{Experimental methods}

One of the most promising novel methods is the artificial ovary. ${ }^{48}$ This innovative technology attempts to generate mature oocytes ready for IVF through a multiphase ex vivo approach, involving simultaneous ovarian, follicle, and oocyte cultures in vitro. ${ }^{54}$ Moreover, techniques like in vitro growth of harvested follicles, oocyte generation using ovarian tissue xenografts in animal models, as well as stem cell (e.g., mesenchymal) utilization, might be available in the future with limited ethical dilemmas. ${ }^{61-64}$

Additionally, understanding of the gonadotoxic mechanisms of commonly used chemotherapeutic agents has enabled the development of preventive adjuvant pharmacotherapy. ${ }^{46}$ There is now a range of agents attempting to protect the ovary against chemotherapy by preventing direct loss and accelerated activation of primordial follicles, as well as follicular atresia. ${ }^{17}$ Although still experimental and only tested in animal models, these methods represent the unique opportunity for fertility preservation in prepubertal leukemia patients with a high risk of ovarian tumor cells. ${ }^{46}$ However, all these regimens still need to be further investigated and developed to be able to protect human ovaries without contralateral damage.

\section{Postpubertal girls and young women}

Although the previously mentioned methods can also be used in postpubertal females, there are few more well-established options with better outcomes that are suitable for this patient group. These include embryo and oocyte cryopreservation, hormonal therapies, and alternative methods such as adoption and oocyte donation.

\section{Embryo and oocyte cryopreservation}

For decades, the preservation of fertilized oocytes has been the gold standard for infertile postpubertal women. With the introduction of cryopreservation of unfertilized oocytes, the cryopreserving technology has also expanded to infertile postpubertal girls and single women. ${ }^{46}$ 


\section{healthbook TIMES Oncology Hematology}

The standard ovulation stimulation protocol, using a combination of gonadotropin-releasing hormone $(\mathrm{GnRH})$ antagonists, gonadotropins, and $\mathrm{GnRH}$ agonists, poses minimal risk of ovarian hyperstimulation syndrome (OHSS) ${ }^{60}$ Stimulation can start at any time during the menstrual cycle. Double stimulation and stimulation directly after ovarian tissue removal are also possible. ${ }^{65-67}$ In a large retrospective analysis, patients with hematological malignancies had a higher number of mature oocytes retrieved; however, the fertilization rate as well as the number of cycles canceled due to OHSS, lack of response to stimulation, and fertilization failure were comparable with other types of cancer. ${ }^{68}$ In cancer patients, the pregnancy rate with frozen embryo per patient was $54.5 \%$, with a subsequent live birth rate of $22.72 \%{ }^{69}$ Comparably, live birth rates in healthy IVF patients were $22-36 \%$. However, there is no specific data of success rate in oocyte cryopreservation for cancer patients, with only $8.36 \%$ of vitrified oocytes resulting in pregnancy in healthy IVF population. ${ }^{70}$

\section{Hormonal therapies}

$\mathrm{GnRH}$ analogs could be prescribed to preserve fertility. However, their role in protecting ovaries before and during chemotherapy in hematological malignancies is controversial. ${ }^{71-73}$ The explanation of their potentially protective function depends on the assumption that the downregulation of pituitary gland will decrease the proliferation rate in the ovaries and result in a lower sensitivity to the cytotoxic agents. ${ }^{74}$ Most meta-analyses provide evidence that temporary ovarian suppression with $\mathrm{GnRH}$ analogs during chemotherapy is an effective and safe option to reduce chemotherapy-induced premature ovarian insufficiency. However, its impact on conception remains to be determined. ${ }^{71,72,75-77}$ Additionally, combination oral contraceptives (COC) represent another hormonal option that may decrease the incidence of ovulation and bleeding during chemotherapy. ${ }^{78}$ A German retrospective study has shown that the COC group had a reduced risk of amenorrhea compared with the women not receiving COC during chemotherapy. ${ }^{39}$ Similarly, a systematic review reported a decreased risk of premature ovarian failure in patients receiving COC. ${ }^{79}$

\section{Alternative methods}

If available fertility preservation methods fail or are not undertaken due to specific reasons, there are still some alternative methods such as adoption and oocyte donation. While surrogacy is currently not legal in Switzerland, patients may travel to countries where this procedure is allowed. ${ }^{80}$ This method is growing on popularity and is called cross border reproductive care. ${ }^{81}$

\section{ONCOFERTILITY APPROACH}

\section{Current challenges}

Hemato-oncologists and reproductive specialists face many challenges in counseling female patients with leukemia about fertility preservation not only due to the anti-cancer treatment but also to the specific patient group and the disease itself.

\section{Urgent treatment}

One of the most frequent challenges in the oncofertility approach is the acute onset of leukemia, which often requires urgent treatment. These patients have no time for fertility preservation procedures since the delay of anti-cancer treatment could endanger their life. ${ }^{60,78}$ Additionally, these patients often present with a poor health condition, including cytopenia, infection, and cardiopulmonary issues, which also makes them ineligible for most fertility preservation procedures. ${ }^{46}$ Postpubertal girls and women could receive GnRH agonists, and COC after risks of accelerated bone loss and thromboembolic events have been carefully calculated, while there is no alternative for prepubertal girls. ${ }^{78}$

\section{Prepubertal age}

Another common problem is that most female patients with leukemia are prepubertal girls with minimal options for fertility preservation. Fortunately, modern chemotherapeutic regimens result in modest fertility impairment; therefore, their fertility might be preserved even if no unique preservation methods are undertaken. ${ }^{1,9}$ Nevertheless, some authors suggest that ovarian tissue cryopreservation should still be performed in girls who can undergo laparoscopic surgery. In the future, this procedure might be routinely used due to the advances in technology. ${ }^{78}$

\section{Unknown gonadotoxic risk}

Counseling on infertility and fertility preservation in leukemia patients can be particularly challenging due to uncertainties in ovarian failure as well as of ovarian recovery rates. Furthermore, the gonadotoxic risks of some novel anti-cancer regimens are still unknown. There is also an undefined risk of some fertility preservation methods that are immediately available.

Therefore, a question may arise if we can overtreat patients with fertility preservation. ${ }^{78}$ In this case, a multidisciplinary approach with fertility counseling and predefined criteria for fertility preservation methods may reduce the uncertainty. 


\section{High risk of relapse}

Most of the modern chemotherapeutic regimens are effective with doses that have a low or very low risk of gonadotoxicity. However, there is a high incidence of relapse in leukemia. In this setting, treatment is intensified, including high-dose chemotherapy and HSCT, which might significantly reduce fertility. Therefore, even after completing therapy, fertility counseling should be undertaken with all patients under the age of 40 years, and further procedures performed depending on the patient's wish. ${ }^{9}$

\section{Chronic myeloid leukemia}

Although CML represents only $0.4 \%$ of all leukemias in females between 15 and 19 years of age, it is especially challenging for fertility management. ${ }^{9}$ The standard of care probably represents a minimal risk to induce infertility; however, it is recommended to consider oocyte or embryo cryopreservation to achieve a timely predicted pregnancy. Namely, due to the potentially harmful and teratogenic effects of treatment with tyrosine kinase inhibitors, the treatment should be interrupted in the time of pregnancy, if the patient has been in a major molecular response for more than two years. Since natural conception can take a while, IVF can limit the off-therapy time and therefore reduces the risk of relapse. ${ }^{47,72,82,83}$ During pregnancy, the tyrosine kinase inhibitors could be exchanged for interferon, whereas some patients with deep molecular response might even stay treatment-free. ${ }^{84}$

\section{Education of hemato-oncologists}

Recent studies have shown that most hemato-oncologists (79\%) and HSCT specialists (87\%) are aware of gonadotoxic risks of anti-cancer treatment and counsel patients about the possibility of infertility. ${ }^{85,86}$ Since reproductive health is not their primary concern, it is recommended that hematooncologists closely collaborate with reproductive specialists and refer patients to fertility counseling at the time of diagnosis. ${ }^{46,60,78}$ However, a recent study showed that only $36 \%$ of female patients were referred to reproductive specialists. ${ }^{85} \mathrm{~A}$ simple discussion about possible risks and benefits has shown substantial improvements in the reduction of stress in patients. $^{87}$ It is essential that we further promote the multidisciplinary approach, which could reduce stress not only for patients but also for the treating hemato-oncologists, who could, therefore, focus more on anti-cancer treatment.
Costs

The additional financial support offered either by the health insurance or by the state, might help increase the number of fertility preservation procedures in leukemia patients. ${ }^{88}$ For example, in the United States, only $3-10 \%$ of cancer patients decide for fertility preservation. The main reason for not providing the oncofertility approach for all suitable patients is the high financial burden. Conversely, in countries like France, where fertility preservation is a statutory right, they reported that $23 \%$ of eligible women with breast cancer had undergone fertility preservation procedures. ${ }^{89}$ In Switzerland, some assisted reproductive techniques have been covered by insurance since July 2019. ${ }^{90}$ However, earlier, the costs of fertility preservation in pediatric and adolescent cancer patients were primarily covered by parents and less frequently by health insurance or organizations, such as the Swiss Cancer League. ${ }^{52}$

\section{Our model for the oncofertility approach}

Numerous guidelines for fertility preservation have been published around the world. ${ }^{46,47,60,78}$ Taking all their aspects and current challenges into consideration, we have designed a model for the oncofertility approach in females with leukemia (Figure 2). We aimed to help hemato-oncologists better understand the current situation and to facilitate the decisions made by reproductive specialists. Although leukemia patients mostly have limited options for fertility preservation, they must be aware of the gonadotoxic effects of anti-cancer treatment, possibly before the beginning of therapy. Therefore, fertility preservation should be routinely discussed with every patient in the reproductive age, and further referrals to specialized oncofertility centers should be undertaken. 


\section{healthbook TIMES Oncology Hematology}

\section{ELIGIBILITY CRITERIA FOR ONCOFERTILITY COUNSELING}

1. Wants fertility preservation

2. Younger than 40 years

3. Favorable prognosis

4. An intermediate or a high-risk gonadotoxic treatment

5. Stable health condition:

- no cytopenia

- no infection

- no cardiopulmonary issues

- no risk of thromboembolism

\section{ELIGIBLE PATIENTS}

Further evaluation of the appropriate fertility preservation methods should follow and additional factors should be considered:

- Costs

- Patients preferences

- Availability of methods

\section{INELIGIBLE PATIENTS}

Invasive fertility preservation methods before anti-cancer treatment should not be undertaken. Recommendations for fertility preservation during anti-cancer treatment for prepubertal and postpubertal patients should be considered:

- Fractionated radiotherapy

- GnRH analogs

- Combination oral contraceptives

Reevaluation after the procedure and the therapy is recommended.

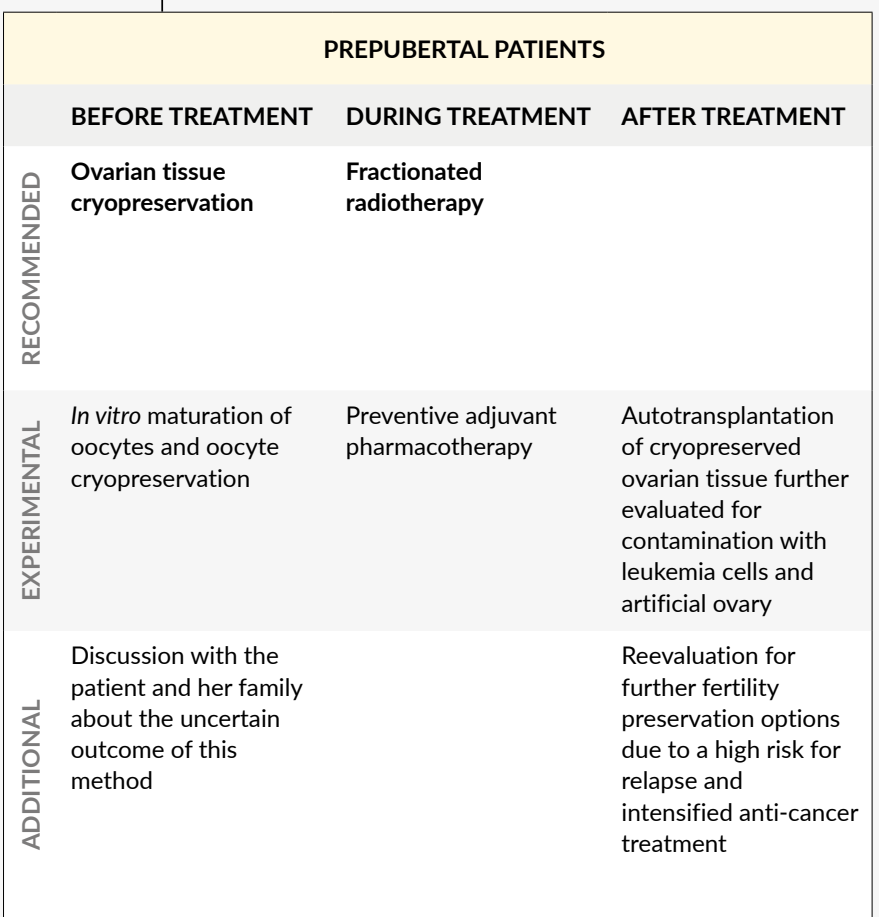

Figure 2. The recommended model for the oncofertility approach in females with leukemia. $\mathrm{GnRH}$, gonadotropin-releasing hormone Adapted from Schüring et al. (2018), Salama et al. (2019), Loren et al. (2019).46,47,78

cryopreservation of
POSTPUBERTAL PATIENTS

\section{BEFORE TREATMENT DURING TREATMENT AFTER TREATMENT}

a) <1 week:

GnRH analogs

b) 2 weeks:

GnRH analogs

Combination oral

contraceptives

$\begin{array}{lll}\text { O Ovarian stimulation } & \text { Fractionated } \\ \text { ₹ } & \text { with oocyte/embryo } & \text { radiotherapy }\end{array}$

एu cryopreservation

c) 3 weeks:

Double ovarian

stimulation with

oocyte/embryo

cryopreservation

Embryo transfer if

natural conception is

not reached

In vitro maturation of Preventive adjuvant

ए oocytes and oocyte

pharmacotherapy

cryopreservation

ovarian tissue

cryopreservation

Autotransplantation of cryopreserved ovarian tissue further evaluated for contamination with leukemia cells and artificial ovary

\section{Additional}

non-fertilized oocytes

for the case that the

couple splits up

Reevaluation for further fertility preservation options due to a high risk for relapse and intensified anticancer treatment 


\section{CONCLUSION}

To mitigate the gonadotoxic risks in girls and young women with leukemia, different fertility preservation methods could be undertaken before, during, and after treatment based on the age of a patient. In order to improve reproductive outcome and quality of life in young cancer survivors, close collaboration between hemato-oncologists with a multidisciplinary oncofertility approach is necessary.

1. Siegel RL et al. Cancer statistics, 2020. CA A Cancer J Clin. 2020; 70(1): 7-30. 2. Leader $\mathrm{A}$ et al. Fertility considerations and preservation in haema-
to-oncology patients undergoing treatment. $\mathrm{Br}$ Haematol. 2011; 153(3): 291-308.

3. Stensheim $\mathrm{H}$ et al. Pregnancy after adolescent and adult cancer: a population-based matched cohort study. Int J Cancer. 2011; 129(5): 1225-36.

4. Speller B et al. Oncofertility Decision Support Resources for Women of Reproductive Age: Systematic Review. JMIR Cancer. 2019; 5(1): e12593.

5. Taylor JF et al. Fertility Preservation after a Cancer Diagnosis: A Systematic Review of Adolescents', Parents', and Providers' Perspectives, Experiences, and Preferences. J Pediatr Adolesc Gynecol. 2016; 29(6): 585-98.

$585-98$.
6. Woodruff TK. Oncofertility: a grand collaboration between repro6. Woodruff TK. Oncofertility: a grand collaboration between
ductive medicine and oncology. Reprod. 2015; 150(3): S1-10. ductive medicine and oncology. Reprod. 2015; 150(3): S1-10.
7. Levine JM et al. Infertility in reproductive-age female cancer survi7. Levine JM et al. Infertility in reproductive-age female cancer survi-
vors: Infertility in Female Cancer Survivors. Cancer. 2015; 121(10): 1532-9.

8. te Velde ER et al. Developmental and endocrine aspects of normal 8. te Velde ER et al. Developmental and endocrine aspects of
ovarian aging. Mol Cell Endocrinol. 1998; 145(1-2): 67-73.

9. Loren AW et al. Fertility preservation in patients with hematologic
ovarian aging. Mol Cell Endocrinol. 1998; $145(1-2): 67-73$. malignancies and recipients of hematopoietic cell transplants. Blood. 2019: 134(9): 746-60.

10. Oktem $\mathrm{O}$ et al. Quantitative assessment of the impact of chemotherapy on ovarian follicle reserve and stromal function. Cancer. 2007; 110(10): 2222-9.

11. Poorvu PD et al. Cancer Treatment-Related Infertility: A Critical Review of the Evidence. JNCI Cancer Spectr. 2019; 3(1): pkz008. 12. Women: risk of amenorrhea from chemotherapy and radiation
treatments for cancer. Fertile Hope 2007. [Accessed June 2020] Available from: http://www.fertilehope.org/healthcare-professionals/clinical-tools/RiskAmenorrhea_v3.pdf.

13. Jacobson $\mathrm{MH}$ et al. Menses resumption after cancer treatment-induced amenorrhea occurs early or not at all. Fertil Steril. 2016; 105(3): $765-72$.

14. Partridge $\mathrm{AH}$ et al. Ovarian reserve in women who remain premenopausal after chemothe

15. Fanchin R. Serum anti-Mullerian hormone is more strongly related to ovarian follicular status than serum inhibin B, estradiol, FSH and LH on day 3. Hum Reprod. 2003; 18(2): 323-7.

16. Dewailly $D$ et al. The physiology and clinical utility of anti-Müllerian hormone in women. Hum Reprod Update. 2014; 20(3): 370-85. 17. Spears $\mathrm{N}$ et al. Ovarian damage from chemotherapy and current approaches to its protection. Hum Reprod Update. 2019; 25(6): 673-93. 18. Luan $Y$ et al. Inhibitors of apoptosis protect the ovarian r
from cyclophosphamide. J Endocrinol. 2019; 240(2): 243-56.

from cyclophosphamide.J Endocrinol. $2019 ; 240(2): 243-56$.
19. Inaba $\mathrm{H}$ et al. Acute lymphoblastic leukaemia. Lancet. 2013; 19. Inaba $\mathrm{H}$ et al. A

381 (9881): $1943-55$.
20oper SL et al. Treatment of Pediatric Acute Lymphoblastic Leu20. Cooper SL et al. Treatment of Pediatric Acute Ly
kemia. Pediatr Clin North Am. 2015; 62(1): 61-73.

kemia. Pediatr Clin North Am. 2015; 62 (1): 61-73.
21. Fey MF et al. Acute myeloblastic leukaemias in adult patients: 21. Fey MF et al. Acute myeloblastic leukaemias in adult patients:
ESMO Clinical Practice Guidelines for diagnosis, treatment and follow-up. Ann Oncol. 2013; 24: vil138-43.

22. Hochhaus A et al. Chronic myeloid leukaemia: ESMO Clinical Practice Guidelines for diagnosis, treatment and follow-up. Ann Oncol. 2017; 28: iv41-51

23. Eichhorst B et al. Chronic lymphocytic leukaemia: ESMO Clinical Practice Guidelines for diagnosis, treatment and follow-up. Ann Oncol. 2015; 26: v78-84.

24. Bellusci G et al. Kinase-independent inhibition of cyclophosphamide-induced pathways protects the ovarian reserve and prolongs fertility. Cell Death Dis. 2019; 10(10): 726

25. Chow EJ et al. Pregnancy after chemotherapy in male and female survivors of childhood cancer treated between 1970 and 1999: a report from the Childhood Cancer Survivor Study cohort. Lancet Oncol. 2016; 17(5): 567-76.

26. Niringiyumukiza JD et al. Protective properties of glycogen synthase kinase-3 inhibition against doxorubicin-induced oxidative damage to mouse ovarian reserve. Biomed Pharmacother. 2019; 116 : 108963.

27. Soleimani R et al. Mechanisms of chemotherapy-induced human ovarian aging: double strand DNA breaks and microvascular compromise. Aging. 2011;3(8): 782-93.

28. Meirow D et al. Toxicity of Chemotherapy and Radiation on Fe-
. 28. Meirow D et al. Toxicity of Chemotherapy and Radiation
male Reproduction: Clin Obstet Gynecol. 2010; 53(4): 727-39. 29. Bedoschi $G$ et al. Chemotherapy-induced damage to ovary: mecha29. Bedoschi $G$ et al. Chemotherapy-induced damage to ovary: me

30. Sukumvanich P et al. Incidence and time course of bleeding after long-term amenorrhea after breast cancer treatment: A prospective long-term amenorrhea after breast cancer
study. Cancer. 2010; 116(13): 3102-11.

study. Cancer. 2010; 116(13): 3102-11.
31. Winship AL et al. Vincristine Chemotherapy Induces Atresia of 31. Winship AL et al. Vincristine Chemotherapy Induces Atresia of 32. Salem W et al. Imatinib treatments have long-term impact on pla-

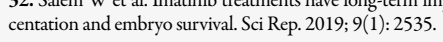

33. Christopoulos $C$ et al. Primary ovarian insufficiency associated with imatinib therapy. N Engl J Med. 2008; 358(10): 1079-80. 34. Cengiz Seval G et al. Gonadotoxic Effects of Nilotinib in Chronic Myeloid Leukemia Treatment Dose in a Mouse Model. Turk J Haematol. 2017; 34(2): 137-42.

35. He $\mathrm{K}$ et al. Lacteal secretion, fetal and maternal tissue distributio of dasatinib in rats. Drug Metab. Dispos. 2008; 36(12): 2564-70. 36. Hutt KJ. The role of $\mathrm{BH}$-only proteins in apoptosis within the ovary. Reprod. 2015; 149(2): R81-9.

37. Waye MM et al. Role of asparaginase synthetase and asparagyl-transfer RNA synthetase in the cell-killing activity of asparaginase in Chinese hamster ovary cell mutants. Cancer Res. 1981; 41(8): 3104-6.

38. Kreuser ED et al. Reproductive and endocrine gonadal functions in adults following multridrug chemotherapy for acute lymphoblastic or in adults following multidrug chemotherapy for acute
undifferentiated leukemia. JCO. 1988; 6(4): 588-95.

39. Behringer Ket al. Secondary Amenorrhea After Hodgkin's Lym39. Behringer Ket al. Secondary Amenorrhea After Hodgkin's Lynphoma Is Influenced by Age at Treatment, Stage of Disease, Chemo-
therapy Regimen, and the Use of Oral Contraceptives During Therapy: therapy Regimen, and the Use of Oral Contraceptives During Therapy: 2005; 23(30): 7555-64.

40. Kim SS et al. Recommendations for fertility preservation in pa40. Kim SS et al. Recommendations for fertility preservation in pa-
tients with lymphoma, leukemia, and breast cancer. J Assist Reprod tients with lymphoma, leuk

Genet. 2012; 29(6): 465-8.
41. Callahan C et al. Pediatric Survivorship: Considerations Following CAR T-Cell Therapy. Clin J Oncol Nurs. 2019; 23(2): 35-41.

CAR T-Cell Therapy. Clin J Oncol Nurs. 2019; 23(2):35-41.
42. Vos MD et al. Fertility preservation in women with cancer. The 42. Vos MD et al. Fertility preservation
Lancet. 2014; 384(9950): 1302-10.

Lancet. 2014; 384(9950): 1302-10.
43. Wallace WHB et al. Predicting age of ovarian failure after radiation 43. Wallace WHB et al. Predicting age of ovarian failure after radiation
to a field that includes the ovaries. Int J Radiat Oncol Biol Phys. 2005; to a field that includ
$62(3): 738-44$

62(3): $738-44$.

44. Wallace WHB et al. The radiosensitivity of the human oocyte. Hum Reprod. 2003; 18(1): 117-21.

45. Wo JY et al. Impact of Radiotherapy on Fertility, Pregnancy, and Neonatal Outcomes in Female Cancer Patients. Int J Radiat Oncol Biol Phys. 2009; 73(5): 1304-12.

46. Salama $\mathrm{M}$ et al. Preserving fertility in female patients with hemaOncol. 2019; 30(11): 1760-75.

47. Schüring AN et al. Practical recommendations for fertility preservation in women by the FertiPROTEKT network. Part I: Indications for fertility preservation. Arch Gynecol Obstet. 2018; 297(1):241-55. 48. Ladanyi $\mathrm{C}$ et al. Recent advances in the field of ovarian tissue cryopreservation and opportunities for research. J Assist Reprod Genet. preservation and opp
2017; 34(6): 709-22.

49. Demeestere I et al. Live birth after autograft of ovarian tissue cryopreserved during childhood: Figure 1. Hum Reprod. 2015; 30(9): 2107-9.

50. Dolmans M-M et al. Risk of transferring malignant cells with 50. Dolmans M-M et al. Risk of transferring malignant cells with
transplanted frozen-thawed ovarian tissue. Fertil Steril. 2013; $99(6)$ : 1514-22.

51. Shapira $M$ et al. First delivery in a leukemia survivor after tran plantation of cryopreserved ovarian tissue, evaluated for leukemia cells contamination. Fertil Steril. 2018; 109(1): 48-53.

52. Diesch $T$ et al. Fertility preservation in pediatric and adolescent cancer patients in Switzerland: A qualitative cross-sectional survey. Cancer Epidemiol. 2016; 44: 141-6.

53. Virant-Klun I et al. Human oocyte maturation in vitro is improved by co-culture with cumulus cells from mature oocytes. Repro Biomed Online. 2018; 36(5): 508-23.

54. Díaz-García $C$ et al. The artificial ovary: any new step is a step forward. Fertil Steril. 2014; 101(4): 940.

55. Silber SJ et al. To transplant or not to transplant - that is the question. Cancer Treat Res. 2010; 156: 41-54

56. Berwanger $\mathrm{AL}$ et al. New trends in female fertility preservation: in vitro maturation of oocytes. Future Oncol. 2012; 8(12): 1567-73. 57. Maman E et al. Luteal phase oocyte retrieval and in vitro maturtion is an optional procedure for urgent fertility preservation. Fertil Steril. 2011;95(1): 64-7.

58. Yang Z-Y et al. Development of in vitro maturation techniques for clinical applications. Fertil Steril. 2017; 108(4): 577-84.

59. Grynberg $M$ et al. First birth achieved after fertility preservation using vitrification of in vitro matured oocytes in a woman with breast cancer. Ann Oncol. 2020; 31(4): 541-2.

60. von Wolff $M$ et al. Practical recommendations for fertility preservation in women by the FertiPROTEKT network. Part II: fertility preservation techniques. Arch Gynecol Obstet. 2018; 297(1): 257-67. 61. Ruan X et al. Randomized study to prove the quality of human
61 ovarian tissue cryopreservation by xenotransplantation into mice. J Ovarian Res. 2019; 12(1): 46

62. Leal ÉSS et al. In vitro growth and development of isolated secondary follicles from vitrified caprine ovarian cortex. Reprod Fertil Dev. 2018; $30(2): 359$.

63. Fazeli Z et al. Mesenchymal Stem Cells (MSCs) Therapy for Recovery of Fertility: a Systematic Review. Stem Cell Rev and Rep. 2018;
$14(1): 1-12$.
64. Dittrich R et al. Xenotransplantation of cryopreserved human ovarian tissue-a systematic review of MII oocyte maturation and discussion of it as a realistic option for restoring fertility after cance treatment. Fertil Steril. 2015; 103(6): 1557-65.

65. von Wolff M et al. Ovarian stimulation to cryopreserve fertilize oocytes in cancer patients can be started in the luteal phase. Fertil Steril. 2009; 92(4): 1360-5.

66. Moffat $R$ et al. Dual ovarian stimulation is a new viable option for enhancing the oocyte yield when the time for assisted reproductive technnology is limited. Reprod Biomed Online. 2014; 29(6): 659-61. 67. Huober-Zeeb C et al. Improving fertility preservation in cancer:
ovarian tissue cryobanking followed by ovarian stimulation can be efficiently combined. Fertil Steril. 2011; 95(1):342-4.

68. Alvarez RM et al. Fertility preservation in female oncology patients: the influence of the type of cancer on ovarian stimulation response. Hum Reprod. 2018; 33(11): 2051-9.

69. Kushnir VA et al. Systematic review of worldwide trends in assisted reproductive technology 2004-2013. Reprod Biol Endocrinol. 2017; 15(1): 6

70. Rienzi L et al. Oocyte, embryo and blastocyst cryopreservation in ART: systematic review and meta-analysis comparing slow-freezing versus vitrification to produce evidence for the development of globa guidance. Hum Reprod Update. 2017; 23(2): 139-55.

71. Hickman LC et al. Preservation of gonadal function in women undergoing chemotherapy: a systematic review and meta-analysis of the potential role for gonadotropin-releasing hormone agonists. J Assist

72. Lambertini $\mathrm{M}$ et al. Gonadotropin-Releasing Hormone Agonists During Chemotherapy for Preservation of Ovarian Function and Fertility in Premenopausal Patients With Early Breast Cancer: A Systematic Review and Meta-Analysis of

73. von Wolff $\mathrm{M}$ et al. Comments on the letter "Fertility preservatio and GnRHa for chemotherapy: debate". Arch Gynecol Obstet. 2010 282(6):717-8.

74. Gründker $\mathrm{C}$ et al. The Role of Gonadotropin-Releasing Hormon in Cancer Cell Proliferation and Metastasis. Front Endocrinol. 2017; 8: 187 .

75. Elgindy E et al. Protecting Ovaries During Chemotherapy Through Gonad Suppression: A Systematic Review and Meta-analysis. Obstet Gynecol. 2015; 126(1): 187-95.

76. Munhoz RR et al. Gonadotropin-Releasing Hormone Agonists for Ovarian Function Preservation in Premenopausal Women Undergoing Chemotherapy for Early-Stage Breast Cancer: A Systematic Review and Meta-analysis. JAMA Oncol. 2016; 2(1): 65-73.

77. Vitek WS et al. Gonadotropin-releasing hormone agonists for the preservation of ovarian function among women with breast cancer who preservation of ovarian function among women with breast cancer who
did not use tamoxifen after chemotherapy: a systematic review and medid not use tamoxifen after chemotherapy: a sy

78. Loren AW. Fertility issues in patients with hematologic malignan78. Loren AW. Fertility issues in patients
cies. Hematology. 2015; 2015(1): 138-45.

cies. Hematology. 2015; 2015(1): 138-45.
79. Blumenfeld $\mathrm{Z}$ et al. GnRH-analogues and oral contraceptives for 79. Blumenfeld $\mathrm{Z}$ et al. GnRH-analogues and oral contraceptives for
fertility preservation in women during chemotherapy. Hum Reprod Update. 2008: 14(6): 543-52

80. No T. CC 810.11 Federal Act of 18 December 1998 on Medically Assisted Reproduction (Reproductive Medicine Act, RMA). Schweizerische Eidgenossenschaft 2017. [Accessed June 2020]. Available from: https://www.admin.ch/opc/en/classified-compilation/20001938/. 81. Salama $\mathrm{M}$ et al. Cross border reproductive care (CBRC): a growing global phenomenon with multidimensional implications (a systematic
and critical review).J Assist Reprod Genet. 2018;35(7): 1277-88. and critical review). J Assist Reprod Genet. 2018; 35(7): 1277-88.
82. Peccatori FA et al. Cancer, pregnancy and fertility: ESMO Clinical Practice Guidelines for diagnosis, treatment and follow-up. Ann Onco 2013; 24: vi160-70.

83. Oktay K et al. Fertility Preservation in Patients With Cancer ASCO Clinical Practice Guideline Update. JCO. 2018; 36(19): 1994-2001.

84. Abruzzese $\mathrm{E}$ et al. Management of pregnant chronic myeloid leukemia patients. Expert Rev Hematol. 2016; 9(8): 781-91.

85. Alexandroni $\mathrm{H}$ et al. Fertility preservation from the point of view of hematopoietic cell transplant specialists-a worldwide-web-base survey analysis. Bone Marrow Transplant. 2019; 54(11): 1747-55. 86. Buske $D$ et al. Die Thematisierung des Kinderwunsches und der Fertilität in der Hämato-Onkologie - eine qualitative Ärztebefragung. Psychother Psych Med. 2015; 65(09/10): 339-44.

87. Logan $S$ et al. Systematic review of fertility-related psychological distress in cancer patients: Informing on an improved model of care Psycho-Oncology. 2019; 28(1): 22-30.

88. Walter JRet al. A Call for Fertility Preservation Coverage for Breast Cancer Patients: The Cost of Consistency. JNCI: Journal of the National Cancer Institute. 2017; 109(5).

89. Phelippeau J et al. Ovarian protection and fertility preservation in 89. Phelippeau J et al. Ovarian protection and fertility preservation in
women with cancer: A French national registry analysis between 2005 women with cancer: A French national registry analysis b

90. Kostenübernahme für fruchtbarkeitserhaltende Massnahmen. Krebsliga Schweiz Juni 2019. [Accessed June 2020]. Available from: https://www.krebsliga.ch/medien/medienmitteilungen/pages/2019/ kostenuebernahme-fuer-fruchtbarkeitserhaltende-massnahmen/. 